\title{
Orígenes e importancia de la Red de Enfermedad de Kawasaki en América Latina (REKAMLATINA)
}

\author{
Documento elaborado por: Antonio González-Mata, Rolando Ulloa-Gutierrez, \\ José Brea del Castillo, Guillermo Soza y Adriana H. Tremoulet
}

Hospital Universitario Pediátrico "Agustín Zubillaga", Barquisimeto, Venezuela (AG-M)

Servicio de Infectología Pediátrica, Hospital Nacional de Niños "Dr. Carlos Sáenz Herrera", San José Costa Rica (RU-G). Centro Médico Universidad Central del Este, Santo Domingo, República Dominicana (JBdC)

Hospital Dr. Hernán Enríquez

Aravena, Temuco, Chile (GS) University of California San Diego/ Rady Children's Hospital San Diego, Estados Unidos de Norteamérica

Fuentes de financiamiento: Ninguna Conflictos de interés: Ninguno que

Recibido: 6 de junio de 2013

Correspondencia a: Antonio González-Mata tonygonz7@yahoo.com
L a enfermedad de Kawasaki (EK) es la causa más común de cardiopatía adquirida en niños de países desarrollados y aquellos en vías de desarrollo que han logrado el control de la fiebre reumática ${ }^{1}$. Esta vasculitis aguda afecta a niños previamente sanos en su inmensa mayoría, ocurriendo $80 \%$ de casos en los primeros cinco años de vida, y cerca de $50 \%$ se presentan en niños bajo dos años de edad. A pesar de que esta enfermedad se describió por primera vez en Japón en la década de los sesentas por Tomisaku Kawasaki ${ }^{2,3}$, su etiología precisa hoy permanece incierta. Sin embargo, se cree que la misma es desencadenada por algún agente infeccioso probablemente viral, y que existe una susceptibilidad genética en algunos individuos a padecer la enfermedad ${ }^{4,5}$.

La epidemiología de la enfermedad ha sido muy bien descrita en Japón y otros países de Asia, Europa, Australia, y Norteamérica ${ }^{6,7}$. Sin embargo, a pesar de que más de veinte países conforman Latinoamérica, a la fecha se desconoce la epidemiología, carga de enfermedad, morbimortalidad y aspectos genéticos en niños de la región. La mayor parte de las descripciones proviene de países aislados, lo constituyen reportes de casos o series pequeñas en su mayoría, o comunicaciones a través de congresos científicos, reuniones de expertos, foros de discusión u otros. La enfermedad no es de declaración obligatoria en los países de la región y existen grandes problemas de diagnóstico y sub-registro de casos. Si bien es cierto que existen varias publicaciones de EK provenientes de países latinoamericanos, la mayor parte de éstas han sido hechas en revistas médicas no indizadas en PubMed, y existen regiones como Centroamérica donde no se conocen datos de algunos países ${ }^{8}$. En Latinoamérica, el estudio poblacional más grande de EK en niños fue recientemente publicado por A. Borzutzky y cols., de Chile, en el año $2012^{9}$. En este estudio se demostró un incremento en las tasas de hospitalización e incidencia de EK en Chile y se propone, al igual que otros investigadores como A. Banfi del Hospital Luis Calvo Mackenna en Santiago lo hicieran en el pasado, la necesidad de un registro nacional de casos de EK y la declaración de ésta como enfermedad de notificación obligatoria.

Motivados por lo anterior, hace unos años un grupo de pediatras infectólogos del Kawasaki Disease Research Center of San Diego, California y de la Sociedad Latinoamericana de Infectología Pediátrica (SLIPE) con gran afinidad, interés y experiencia en EK, vio la necesidad de conocer la epidemiología de EK en niños de Latinoamérica. La carga de la enfermedad, la morbi-mortalidad asociada, y sus posibles repercusiones en la salud de los niños adolescentes o adultos jóvenes a largo plazo, ha sido motivo de preocupación para estos expertos. Por lo tanto, diferentes actividades académicas, reuniones de expertos, foros de discusión e intercambios de opinión se han llevado entre los acá firmantes y otros colegas de la región.

El tema de EK había sido discutido previamente en pocos congresos, jornadas o capítulos regionales de la SLIPE. Sin embargo, durante el XIII Congreso Latinoamericano de Infectología Pediátrica (SLIPE 2009) realizado en Guayaquil, Ecuador, Antonio González-Mata de Venezuela impartió la conferencia plenaria "Enfermedad de Kawasaki: estado del arte”. En la misma se realizó una descripción de la enfermedad en general, así como datos epidemiológicos de países selectos, y se mencionó la ausencia de datos en varios países de Latinoamérica.

A mediados del 2010, se planeó la realización de un 
simposio sobre EK para el XIV Congreso de SLIPE a realizarse en mayo de 2011 en Punta Cana, República Dominicana. En diciembre de ese mismo año, Rolando Ulloa-Gutiérrez realizó una visita al Kawasaki Disease Research Center of San Diego, California, donde se reunió con las pediatras infectólogas Jane C Burns y Adriana $\mathrm{H}$ Tremoulet. Esta última, fue la promotora inicial de la necesidad de establecer una red para la región. Durante dicha visita se discutió la situación epidemiológica actual de EK en Latinoamérica, la necesidad de conocer y dar a conocer los datos de cada país y de la región. Se establecieron ideas para futuros proyectos, la necesidad de trabajar en la red y se afinaron detalles para el Simposio de EK en el seno del congreso de SLIPE de mayo de 2011.

Durante este último se realizó un encuentro bajo la modalidad de "Desayuno con el experto" con los Dres. Tremoulet y Ulloa-Gutiérrez. El mismo contó con una nutrida participación de médicos de la región. De igual forma, se realizó el primer Simposio de Enfermedad de Kawasaki durante un SLIPE y en el cual participaron como conferencistas A. Tremoulet, R. Ulloa-Gutiérrez, G. Soza (Chile) y A. González-Mata. Durante el mismo se abordaron los problemas de diagnóstico, diagnóstico diferencial y sub registro de casos en la región, así como tratamiento convencional y nuevas opciones para casos refractarios, epidemiología de la EK en América Latina, y se reiteró la necesidad de un registro nacional de casos por país así como la notificación obligatoria la enfermedad. Así mismo, se discutieron propuestas de investigaciones futuras, dentro de las cuales la más sobresaliente fue la estructuración de una red latinoamericana de investigadores en EK.

En febrero de 2012, se realizó el $10^{\circ}$ Simposio Internacional de Enfermedad de Kawasaki (ISKD) en Kyoto, Japón. Este es el congreso científico más importante de esta enfermedad en el mundo y en el mismo convergen investigadores principalmente de Japón, Asia, Norte América y Europa. Durante la sesión de presentaciones orales de epidemiología por países, R. Ulloa-Gutiérrez expuso la epidemiología de EK en Costa Rica y comentó la idea del grupo de expertos de crear una red latinoamericana para $\mathrm{EK}^{10}$. Durante dicho congreso, la ausencia de datos de América Latina y presencia de un único país de Latinoamérica en el evento fue discutida por este investigador así como por Jane W. Newburger (Boston, Mass.) en su sesión de clausura y resumen del congreso.

En abril de 2012, en México D.F. Patricia Saltigeral, A. Tremoulet, y R. Ulloa-Gutiérrez discutieron la necesidad de integración en la red de países con poblaciones grandes y numerosos hospitales de México, Brazil, Argentina, Chile, Colombia y Venezuela, así como otros aspectos de logística y planes futuros. En México, por ejemplo, recientemente se ha conformado una red nacional de investigadores en EK para mejorar la vigilancia y re- gistro de casos de la enfermedad ${ }^{11}$. En diciembre de ese mismo año, durante el XXXI Congreso Interamericano de Infectología Pediátrica en Aguas Calientes, México, se reunieron A. Tremoulet y José Brea del Castillo de República Dominicana y Presidente de SLIPE 2011-2013, quienes propusieron que se desarrollara un material educativo enfocado al diagnóstico y tratamiento de EK para la página de internet de la SLIPE, entre otras actividades.

Finalmente, los días 18 y 19 abril de 2013 se llevó a cabo una reunión de trabajo convocada por la SLIPE en La Romana, República Dominicana. En la misma, participaron J. Brea, A. Tremoulet, R. Ulloa-Gutiérrez y A. González-Mata, y en ésta se discutieron numerosos puntos en torno a la creación de la red, la necesidad de estudios de investigación y publicaciones científicas. Se acordó nombrar a dicha red, con el acrónimo REKAMLATINA, Red de Enfermedad de Kawasaki en América Latina. Durante dicha reunión se estableció la lista de países por participar en la red así como los proyectos de investigación prospectivos y retrospectivos durante el presente y siguientes años. Se presentó el proyecto y la logística del mismo ante la mesa directiva de la SLIPE período 20112013. Su presidente, José Brea, ha dado particular apoyo a la idea de establecer dicha red. Se acordó una reunión técnica de trabajo para el próximo Congreso XV de SLIPE en Sao Paulo, Brasil del 26 al 29 de junio 2013, donde entre otros aspectos, se discutirán los aspectos logísticos de la red y del proyecto de investigación "Estudio prospectivo multicéntrico multinacional de la epidemiología de enfermedad de Kawasaki en niños de América Latina"*.

Es así como REKAMLATINA nace e inicia un histórico camino para el conocimiento de la epidemiología de la EK en niños de México, Guatemala, Honduras, El Salvador, Nicaragua, Costa Rica, Panamá, Cuba, República Dominicana, Puerto Rico, Colombia, Venezuela, Ecuador, Perú, Bolivia, Brasil, Paraguay, Uruguay, Chile y Argentina. Hoy, REKAMLATINA se constituye como la red internacional multinacional en el mundo con el mayor número de países integrados para la investigación de la epidemiología de la enfermedad de Kawasaki en niños. Más de 120 investigadores provenientes de más de 85 hospitales donde se hospitalizan y tratan la mayoría de niños con EK en 20 países de Latinoamérica, contribuirán al mejor entendimiento de la epidemiología de la enfermedad en nuestra región. La historia del nacimiento de esta red y el futuro impacto que tenga en la salud de la niñez latinoamericana y de otros países del mundo, será motivo de inspiración para otros países del orbe. Es así en esta revista, como se escriben las páginas de la historia de enfermedad de Kawasaki en América Latina.

\footnotetext{
* Nota del Editor: reunión llevada a cabo el 28 de junio de 2013 en Sao Paulo, Brasil.
} 


\section{Resumen}

La enfermedad de Kawasaki representa la causa más común de cardiopatía adquirida en niños. Si bien es cierto que la epidemiología de la enfermedad ha sido muy bien descrita en Japón y otros países de Asia, Europa, Australia y Norte América, se desconoce la epidemiología y carga de enfermedad en niños de América Latina. Por esta razón nació la idea de establecer una red de investigación de enfermedad de Kawasaki en niños de América Latina, constituyéndose ésta en la red internacional multinacional más grande de investigación de la epidemiología de la enfermedad y donde se integran 20 países de la región.

\section{Referencias bibliográficas}

1.- Newburger J W, Takahashi M, Gerber M A, Gewitz M H, Tani L Y, Burns J C, et al. Committee on Rheumatic Fever, Endocarditis, and Kawasaki Disease, Council on Cardiovascular Disease in the Young, American Heart Association. Diagnosis, treatment, and long-term management of Kawasaki disease: a statement for health professionals from the Committee on Rheumatic Fever, Endocarditis, and Kawasaki Disease, Council on Cardiovascular Disease in the Young, American Heart Association. Pediatrics 2004; 114: 1708-33.

2.- Kawasaki T. MCLS- Clinical observation of 50 cases. Jap J Allerg 1967; 16: 178.

3.- Kawasaki T, Kosaki F, Okawa S, Shigematsu I, Yanagawa H. A new infantile acute febrile mucocutaneous lymphnode syndrome (MLNS) prevailing in Japan. Pediatrics 1974; 54: 271-6.

4.- Khor C C, Dávila S, Breunis W B, Lee Y C, Shimizu C, Wright V J, et al. Genome-wide association study identifies FCGR2A as a susceptibility locus for Kawasaki disease. Nat Genet 2011; 43: 1241-6.

5.- Khor C C, Dávila S, Shimizu C, Sheng S, Matsubara T, Suzuki Y, et al. Genome-wide linkage and association mapping identify susceptibility alleles in ABCC4 for Kawasaki disease. J Med Genet 2011; 48: 467-72.

6.- Uehara R, Belay E D. Epidemiology of Kawasaki disease in Asia, Europe, and the United States. J Epidemiol 2012; 22: 79-85.

7.- Salo E, Griffiths E P, Farstad T, Schiller B, Nakamura Y, Yashiro M, et al. Incidence of Kawasaki disease in northern European countries. Pediatr Int 2012; 54: 770-2.

8.- Ulloa-Gutiérrez R, Alegría M, Estripeaut D, Gamero M, Rodríguez F J, Hernández de Mezerville M, et al. Lack of reports and publications on Kawasaki disease in children from Central America, period 2000-2010.

En: $7^{\text {th }}$ World Congress of the World Society for Pediatric Infectious Diseases (WSPID). Melbourne, Australia. 19 Noviembre 2011. Poster 14

9.- Borzutzky A, Hoyos-Bachiloglu R, Cerda J, Talesnik E. Rising hospitalization rates of Kawasaki Disease in Chile between 2001 and 2007. Rheumatol Int 2012; 32: 2491-5.

10.- Ulloa-Gutiérrez R. Kawasaki syndrome in Costa Rican children. En: The $10^{\text {th }}$ International Kawasaki Disease Symposium (IKDS). February 7-10, 2012. Kyoto, Japón. Presentación/Abstract O-4.

11.- Coria Lorenzo J J. Enfermedad de Kawasaki. Grupo regional para la vigilancia y prevención de enfermedad de Kawasaki en México. Rev Enferm Infecciosas 2012; XXVII: 213-4. 